\title{
BMJ Open Test accuracy of drug and antibody assays for predicting response to antitumour necrosis factor treatment in Crohn's disease: a systematic review and meta-analysis
}

Karoline Freeman, ${ }^{1}$ Sian Taylor-Phillips, ${ }^{1}$ Martin Connock, ${ }^{1}$ Rachel Court, ${ }^{1}$
Alexander Tsertsvadze, ${ }^{1}$ Deepson Shyangdan, ${ }^{1}$ Peter Auguste, ${ }^{1}$ Hema Mistry, ${ }^{1}$
Ramesh Arasaradnam, ${ }^{1,2}$ Paul Sutcliffe,,${ }^{1}$ Aileen Clarke ${ }^{1}$
To cite: Freeman K, TaylorPhillips S, Connock M, et al. Test accuracy of drug and antibody assays for predicting response to antitumour necrosis factor treatment in Crohn's disease: a systematic review and meta-analysis. BMJ Open 2017;7:e014581. doi:10.1136/ bmjopen-2016-014581

- Prepublication history and additional material are available. To view these files please visit the journal online (http://dx.doi. org/10.1136/bmjopen-2016014581).

Received 19 October 2016 Revised 9 February 2017 Accepted 4 April 2017

\section{CrossMark}

${ }^{1}$ Warwick Medical School, University of Warwick, Coventry, Warwickshire, UK

${ }^{2}$ Department of

Gastroenterology, University

Hospital Coventry and

Warwickshire, Coventry, UK

Correspondence to

Sian Taylor-Phillips; s.taylor-

phillips@warwick.ac.uk

\section{ABSTRACT}

Objective To present meta-analytic test accuracy estimates of levels of antitumour necrosis factor (anti-TNF) and antibodies to anti-TNF to predict loss of response or lack of regaining response in patients with anti-TNF managed Crohn's disease.

Methods MEDLINE, Embase, the Cochrane Library and Science Citation Index were searched from inception to October/November 2014 to identify studies which reported $2 \times 2$ table data of the association between levels of anti-TNF or its antibodies and clinical status. Hierarchical/bivariate meta-analysis was undertaken with the user-written 'metandi' package of Harbord and Whiting using Stata V.11 software, for infliximab, adalimumab,anti-infliximab and anti-adalimumab levels as predictors of loss of response. Prevalence of Crohn's disease in included studies was meta-analysed using a random effects model in MetaAnalyst software to calculate positive and negative predictive values. The search was updated in January 2017.

Results 31 studies were included in the review. Studies were heterogeneous with respect to the type of test used, criteria for establishing response and loss of response, population examined and results. Meta-analytic summary point estimates for sensitivity and specificity were $65.7 \%$ and $80.6 \%$ for infliximab trough levels and $56 \%$ and $79 \%$ for antibodies to infliximab, respectively. Pooled results for adalimumab trough levels and antibodies to adalimumab were similar. Pooled positive and negative predictive values ranged between $70 \%$ and $80 \%$ implying that between $20 \%$ and $30 \%$ of both positive and negative test results may be incorrect in predicting loss of response. Conclusion The available evidence suggests that these tests have modest predictive accuracy for clinical status; direct test accuracy comparisons in the same population are needed. More clinical trial evidence from test-treat studies is required before the clinical utility of the tests can be reliably evaluated.

\section{INTRODUCTION}

Antitumour necrosis factor (anti-TNF $\alpha$ ) agents, including infliximab (Remicade, Merck
Strengths and limitations of this study

- This is the first study to summarise predictive accuracy of tests for loss of response to antitumour necrosis factor drugs for managing Crohn's disease, in a clinically relevant manner.

- We included more studies than previous metaanalyses.

- We investigated drug and antibody levels for both infliximab and adalimumab.

- Many of the included studies had a high risk of bias.

- There was insufficient data for subgroup analyses for some types of test.

Sharp \& Dohme) and adalimumab (Humira, AbbVie), are well-established second-line or third-line therapies for people with Crohn's disease $(\mathrm{CD})$. Failure to respond during induction therapy and loss of response (LOR) after initial success are widely documented. ${ }^{1-5}$ One suggested mechanism for this is the production of antibodies which neutralise the anti-TNF $\alpha$ agents and hasten their clearance from the circulation, thus reducing drug availability. The treatment strategy for LOR is usually to escalate the drug dosage or to shorten the dosage interval. If this fails, a switch to an alternative anti-TNF agent can be tried in order to minimise the influence of antidrug antibodies directed against the first agent. Another suggested underlying mechanism for LOR is that cytokines other than TNF $\alpha$ may become the major inflammatory agents. This suggestion arises from the observation that some patients have a LOR to anti-TNF despite the presence of therapeutic drug levels and an absence of anti-TNF antibodies. For such patients, the continued use of anti-TNFs may be considered futile and a switch to different 
biological therapies or other agents may represent the preferred strategy.

The potential role of anti-TNF antibodies and of subtherapeutic drug levels in LOR has provided the impetus for the development of assays for both anti-TNF drugs and for antibodies, and a plethora of studies using such assays have been produced, exploring the association between either levels of antibodies to anti-TNF agents and clinical response or levels of drugs and clinical response. Studies have measured LOR to the administered anti-TNF agent or failure to regain response after a change in treatment. By dichotomising the outcomes at various detectable levels of drug and of antibodies to anti-TNF, the diagnostic value of these tests in predicting LOR or lack of regaining response has been assessed.

Several authors have meta-analysed studies which have reported the association between levels of antibodies to anti-TNF agents and clinical status. ${ }^{6-9}$ These authors have presented pooled relative risk or odds ratio (OR) statistics for clinical state (eg, response or LOR) investigating positive versus negative test result patients (ie, antibodies to anti-TNF agent present or absent), or conversely for test result (positive or negative) in patients with response versus those without response. Although these pooled statistics provide useful information on the association between antibody levels and clinical status, they do not address the question of test accuracy when tests are used as a predictor of patients' clinical response status which is the perspective likely to be adopted by clinicians for patients receiving treatment that may be predicated on test results. Primary studies frequently report test accuracy analysis such as receiver operating characteristic (ROC) curves and test accuracy measures such as sensitivity and specificity. When viewed as diagnostic tests, ${ }^{10}$ it becomes possible to perform alternative meta-analysis so as to obtain pooled estimates of test accuracy. The predictive accuracy of such tests is of considerable practical interest. Our objective therefore is to present the meta-analytic results in terms of pooled test accuracy estimates. A particular advantage of this method is that it allows for investigation of the covariance of associations or, from the perspective of a predictive test, the covariance between sensitivity and specificity, thus giving a more complete picture of the value of these tests in clinical practice.

\section{METHODS}

\section{Search for studies}

An iterative procedure was used to develop the initial MEDLINE search, which was subsequently adapted appropriately for other databases and online resources. We searched multiple bibliographic databases including MEDLINE, Embase, the Cochrane Library and Science Citation Index from inception to October/November 2014. Searches of other online resources including trial registries were also undertaken. Full details of the search strategies used, with exact search dates, are provided in the online supplement 1 . Reference lists of included studies and relevant review articles were checked. Citation searches of selected included studies were undertaken. An update of the search was undertaken in January 2017 (see online supplement 2 figure 1 and table 1 ) .

\section{Study eligibility criteria}

We included studies of patients with $\mathrm{CD}$ treated with infliximab or adalimumab. Studies with mixed Crohn's and ulcerative colitis populations were included if the proportion of Crohn's patients was at least $70 \%$. The intervention of interest was a test measuring serum anti-TNFo (infliximab or adalimumab) and/or anti-infliximab or anti-adalimumab antibody levels. Studies reporting clinical status (ie, response or lack of response) as an outcome were eligible for inclusion. The reported results had to allow for cross-tabulation of dichotomous test outcome with clinical status by means of $2 \times 2$ tables in order to calculate the diagnostic test accuracy parameters. All primary study designs were included.

\section{Study selection}

Two reviewers independently assessed titles and abstracts for inclusion using a prepiloted form. All potentially relevant publications were retrieved and examined independently. Any disagreements regarding inclusion/exclusion were discussed and resolved with a third reviewer. The study selection process and reasons for exclusion at full text screening level are presented in the PRISMA study flow diagram (see figure 1).

\section{Quality assessment}

Studies were quality assessed using a modified QUADAS-2 checklist. ${ }^{11}$ Items included were method of patient selection, blinding of index test results, exclusion of uninterpretable test results from $2 \times 2$ table data and method of assessment of clinical status (the reference case).

\section{Evidence synthesis and statistical methods}

Patient numbers within extracted $2 \times 2$ data tables were used to generate Forest plots of paired sensitivity and specificity (accompanied by 95\% CIs) using Review Manager (RevMan V.5.1; Nordic Cochrane Centre, Copenhagen, Denmark) for four different tests: (1) infliximab levels as predictor of loss of or lack of regaining response, (2) antibodies to infliximab as predictor of loss of or lack of regaining response, (3) adalimumab levels as predictor of loss of or lack of regaining response and (4) antibodies to adalimumab as predictor of loss of or lack of regaining response. Hierarchical/bivariate ${ }^{12}$ meta-analysis was undertaken with the user-written 'metandi' package of Harbord and Whiting ${ }^{13}$ using Stata V.11 software. Positive and negative predictive values were calculated ${ }^{14}$ at the pooled prevalence of LOR in the test population. Prevalence was meta-analysed using a random effects model in MetaAnalyst software. ${ }^{15}$ For meta-analyses which incorporated 10 or more studies, we examined the risk of publication bias (see online supplement 3) mindful of the caveats relating to this in diagnostic test accuracy studies. ${ }^{16}$ 


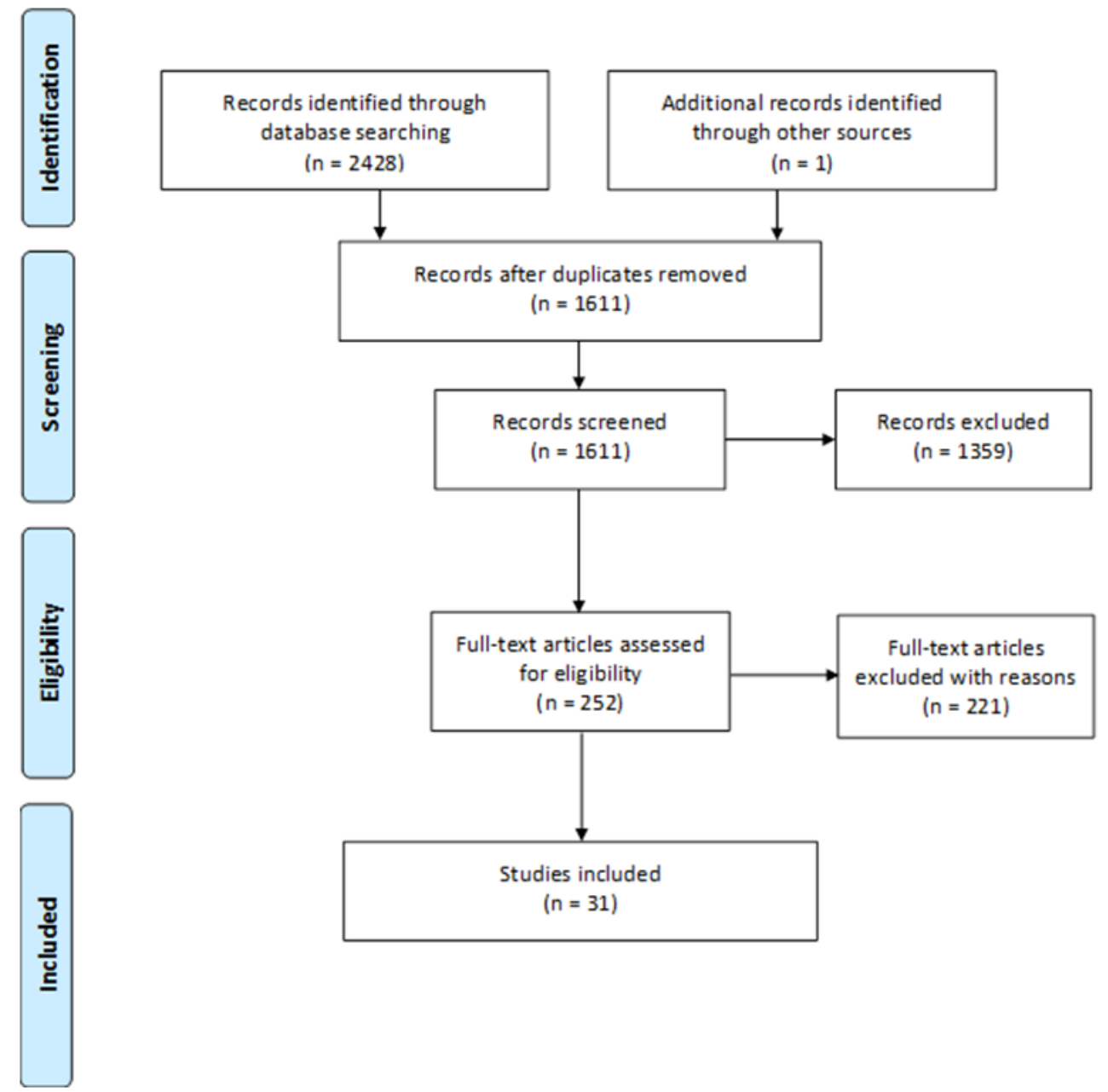

Figure 1 PRISMA study flow diagram.

The protocol for this review was registered on PROSPERO 2014:CRD42014015278. The full protocol is included in the online supplementary appendix 1 .

\section{RESULTS}

We identified 2429 records of which 31 were eligible for inclusion (see online supplement 4 table 1 for excluded studies with reason). Of these, 24 were full-text reports and 7 were conference abstracts. The PRISMA flow diagram is detailed in figure 1 . Eleven of the 31 studies examined infliximab trough levels, 20 examined levels of antibodies to infliximab and five and six studies, respectively, investigated adalimumab levels and antibodies to adalimumab (table 1). The range of anti-TNF cut-offs used for the dichotomisation of test outcomes is illustrated in the online supplement 5 tables 1-3). The risk of bias of studies varied. The greatest threat to validity was high risk of bias in patient selection, for example, studies did not enrol a consecutive or randomly selected patient group. This was present in nearly $80 \%$ of included studies (see online supplement 6 table 1 and figure 1).

The studies were heterogeneous with respect to the type of test used (eg, commercial or in-house ELISA, radioimmunoassay (RIA), homogeneous mobility shift assay (HMSA)), criteria for establishing response or lack of regaining response (eg, use of the Crohn's Disease Activity Index score or the physician's global assessment score) and population examined (responders or patients with secondary loss of response). Sensitivity and specificity pairs are summarised in figure 2 for antibodies to anti-TNF and figure 3 for anti-TNF trough levels.

The paired forest plots show that sensitivity and specificity of using anti-TNFs or antibodies produced against anti-TNFs to predict response or LOR vary greatly among studies with sensitivity revealing generally greater variation. Sensitivity analysis suggests that assay type may explain some of the variation in results between studies of anti-infliximab antibodies; however, there was considerable heterogeneity between numerous study covariates (population, assay type, response criterion), and we do not know whether these might fully explain the large differences in results between studies.

\section{Infliximab trough level tests for LOR or lack of regaining response}

Of 11 included studies, 2 were reported only as abstracts (Ben-Basset et $a l^{17}$ and Yanai et $a l^{18}$ ). The meta-analysis (figure 4) yielded a pooled summary point of $66 \%$ 
Open Access

Table 1 Major features of studies included for hierarchical meta-analyses

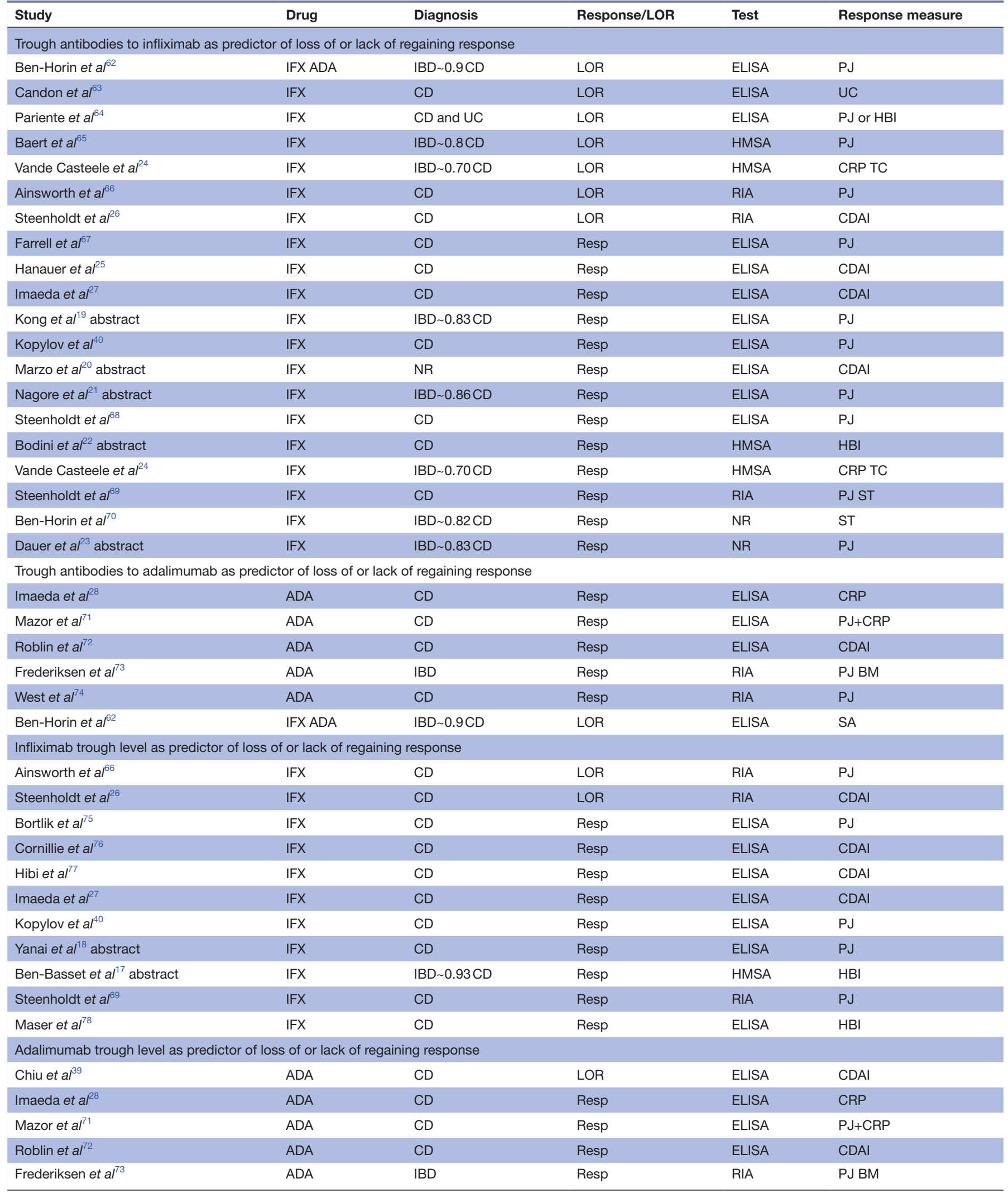

ADA, adalimumab; CD, Crohn's disease; CDAl, Crohn's Disease Activity Index score; CRP, C reactive protein level; Diagnosis, study patient population; HBI, Harvey Bradshaw Index score; HMSA, homogenous mobility shift assay; IBD, inflammatory bowel disease; IFX, infliximab; LOR, patients with loss of response; NR, not reported; PJ, physicians' judgement; PJ BM, physicians' judgement and biological measure; Resp, responding patients; Response measure, method used for defining clinical response; RIA, radioimmunoassay; SA, switch anti-TNF; ST, stop anti-TNF; TC, treatment change. 


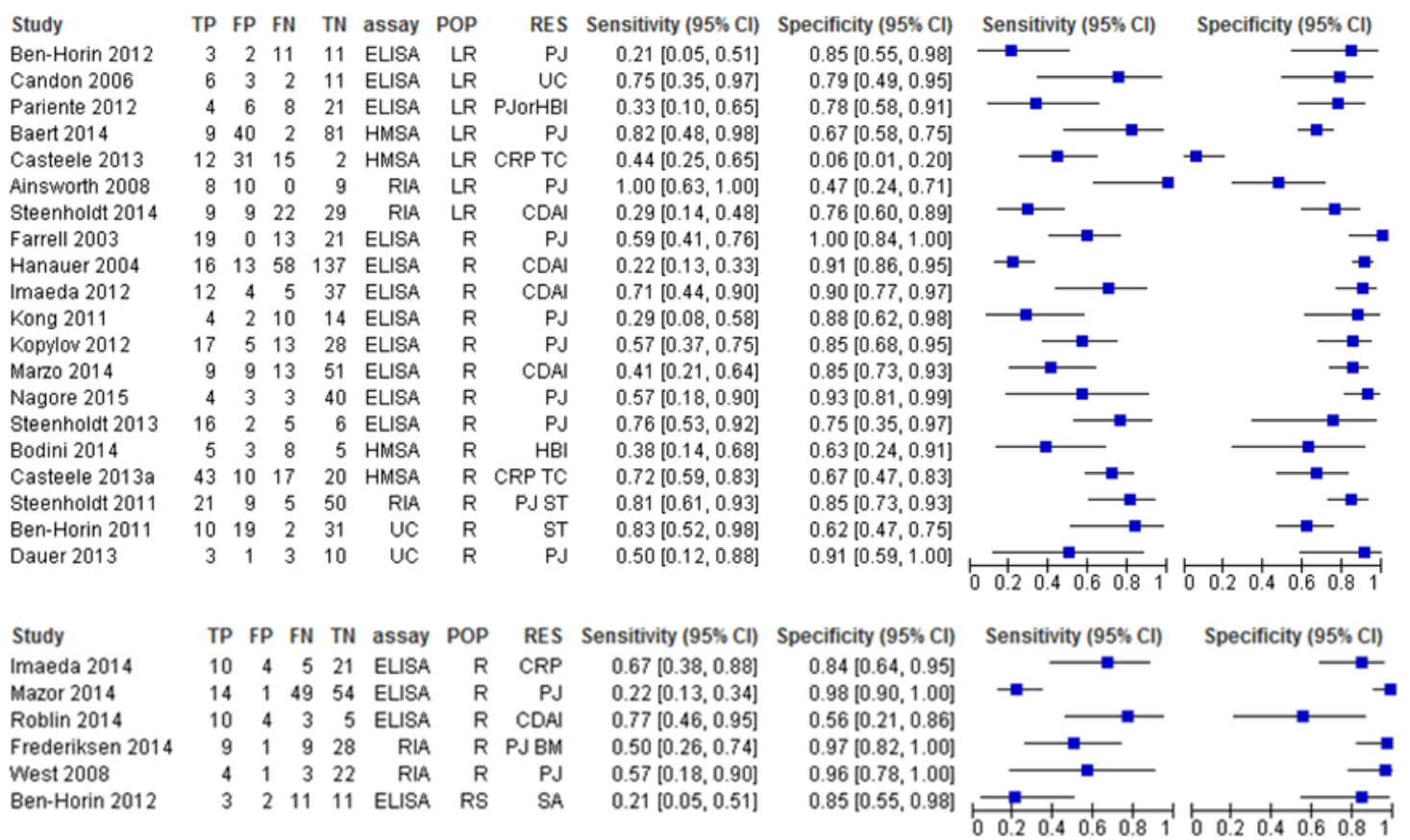

Figure 2 Paired forest plots for anti-TNF antibody levels for predicting loss of response or failure to regain response to infliximab (top) and adalimumab (bottom). CDAI, Crohn's Disease Activity Index score; CRP, C reactive protein level; $\mathrm{HBI}$, Harvey Bradshaw Index score; HMSA, homogeneous mobility shift assay; LR, patients with loss of response; PJ, physicians' judgement; PJ BM, physicians' judgement and biological measure; POP, study patient population; R, patients with response; RES, criterion for determining clinical response; RIA, radioimmunoassay; RS, restart anti-TNF after drug holiday; SA, switch anti-TNF; ST, stop anti-TNF therapy; TC, treatment change; UC, unclear.

sensitivity and $81 \%$ specificity (other test accuracy statistics are summarised in the online supplement 3 ). Sensitivity analysis in which only studies of responder populations were included generated very similar results as did analysis that only included studies with ELISA tests.

\section{Antibodies to infliximab tests for LOR or lack of regaining response}

Of 20 included studies, 5 were reported as abstracts. ${ }^{19-23}$ Sensitivity and specificity pairs are summarised in figure 5 . The pooled summary points for sensitivity and specificity were $56 \%$ and $79 \%$, respectively (figure 5 ). Only minor differences were introduced in the test accuracy outcomes (eg, $60 \%$ and $81 \%$ for sensitivity and specificity, respectively) in a sensitivity analysis when two influential studies were omitted from the analysis. ${ }^{24} 25$ Sensitivity analyses in which only responder studies were included had little effect. Sensitivity analysis in which only ELISA studies were included showed an improvement in specificity at the expense of sensitivity and a

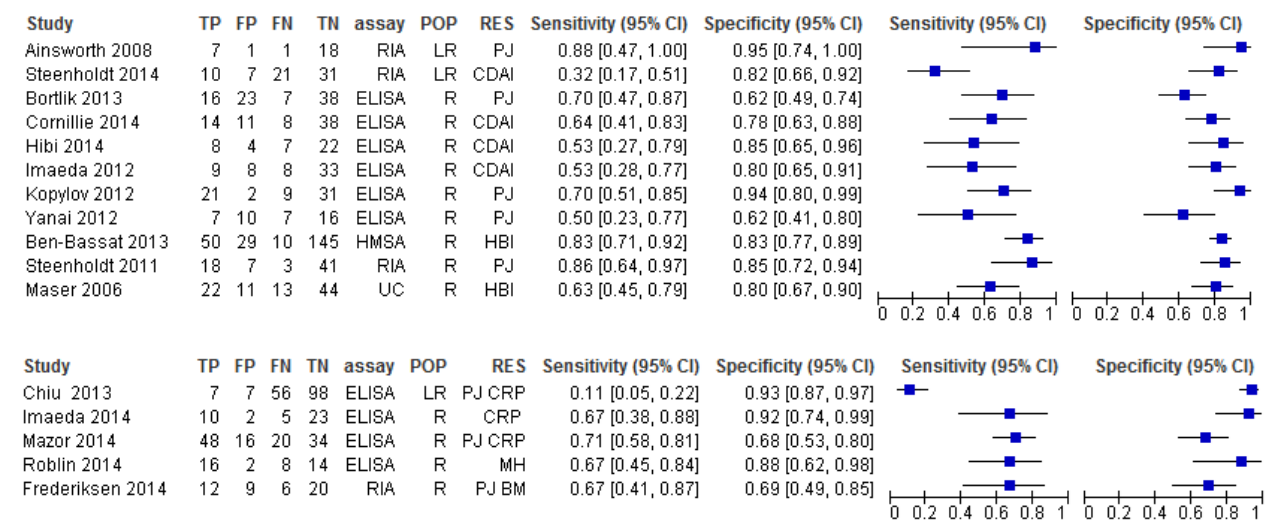

Figure 3 Paired forest plots for trough anti-tumour necrosis factor levels for predicting loss of response or failure to regain response to infliximab (top) and adalimumab (bottom). CDAl, Crohn's Disease Activity Index score; CRP, C reactive protein level; HBI, Harvey Bradshaw Index score; HMSA, homogeneous mobility shift assay; LR, patients with loss of response; MH, mucosal healing; PJ, physicians' judgement; PJ BM, physicians' judgement and biological measure; POP, study patient population; R, patients with response; RES, criterion for determining clinical response; RIA, radioimmunoassay; UC, unclear. 

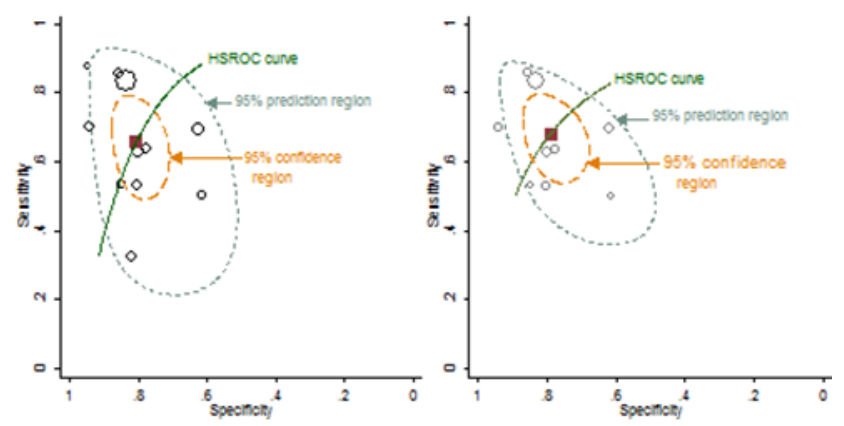

Figure 4 Hierarchical meta-analysis of trough infliximab levels for predicting loss of response or failure to regain response. Left, all 11 studies; right, responder studies only $(n=9)$. The square symbol represents the summary point estimate on the hierarchical summary receiver operating characteristic (HSROC) curve.

reduction in the heterogeneity of specificity measurements (figure 5).

\section{Adalimumab or anti-adalimumab antibody levels as tests for LOR or lack of regaining response}

Far fewer studies of adalimumab-treated patients were available compared with infliximab (table 1). Meta-analysis of patients treated with adalimumab yielded slightly lower test accuracy statistics with wider uncertainty around them compared with those found for infliximab studies (see online supplement 7 table 1 and figure 1 ).

\section{Combined assessment of anti-TNF levels and antibodies to anti-TNF}

Three independent studies reported both drug and antibody test results by individual in relation to the individual's clinical status, response/LOR ${ }^{26} 27$ or regaining response/not regaining response. ${ }^{28}$ These studies allowed calculation of the number of patients in each of the two clinical states distributed to each of the four possible combinations of test result. ${ }^{26-28}$ The results summarised in tables 2 and 3 indicate the probability of LOR to anti-TNF, and table 4 summarises the probability of not regaining response to infliximab according to each possible test result category. These test results are reasonably similar to those from our meta-analysis of single test studies. This comparison should be viewed in the light of the considerable uncertainty which exists because of the small number of studies measuring both drug and antibody levels in the same individuals and their small size.

\section{Predictive values of drug and antidrug antibody tests for LOR or failure to regain response}

In the Cochrane Handbook for Systematic Reviews of Diagnostic Test Accuracy, Bossuyt et al (2013) ${ }^{14}$ suggest that predictive values are more widely and readily

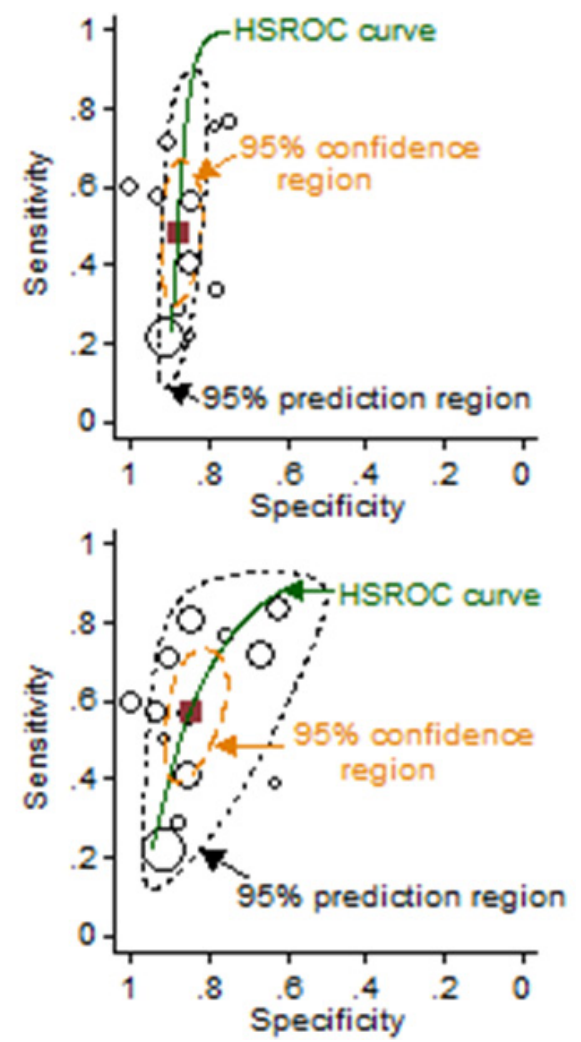

Figure 5 Hierarchical meta-analysis of trough levels of antibodies to infliximab for predicting loss of response or failure to regain response. Top left, all 20 studies; top right, ELISA studies only $(n=9)$; lower left, all studies minus two influential studies $(n=18)^{26} 66$; lower right, responder studies only $(n=13)$. The square symbol represents the summary point estimate on the hierarchical summary receiver operating characteristic (HSROC) curve. 
Table 2 Combined assessment of adalimumab and antiadalimumab levels for responders receiving adalimumab

\begin{tabular}{|c|c|c|c|c|}
\hline $\begin{array}{l}\text { Imaeda } \\
\text { et }\left.a\right|^{28}\end{array}$ & ADAbs+ & ADAbs- & Total & $\begin{array}{l}\text { Population anc } \\
\text { anti-TNF } \alpha \\
\text { therapy; tests }\end{array}$ \\
\hline \multirow{2}{*}{$\begin{array}{l}\text { Anti- } \\
\text { TNF } \alpha-\end{array}$} & Ler & 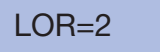 & LUT & \multirow{6}{*}{$\begin{array}{l}\text { Responders on } \\
\text { adalimumab } \\
\text { maintenance; } \\
\text { ELISA. } \\
\text { Prevalence of } \\
\text { LOR=37.5\% }\end{array}$} \\
\hline & $\mathrm{RESP}=0$ & $\mathrm{RESP}=2$ & $\mathrm{RESP}=2$ & \\
\hline \multirow{4}{*}{$\begin{array}{l}\text { Anti- } \\
\text { TNF } \alpha+ \\
\text { Total }\end{array}$} & $\mathrm{LOR}=2$ & $\mathrm{LOR}=3$ & $\mathrm{LOR}=5$ & \\
\hline & $\mathrm{RESP}=4$ & $\mathrm{RESP}=19$ & $\mathrm{RESP}=23$ & \\
\hline & LOF & LOF & LOR & \\
\hline & $\mathrm{RESP}=4$ & $\mathrm{RESP}=21$ & $\mathrm{RESP}=25$ & \\
\hline
\end{tabular}

The probability of a patient returning each of the four possible test result combinations was:

ADAbs+/anti-TNF $\alpha-, 0.200$; ADAbs+/anti-TNF $\alpha+, 0.150$; ADAbs-/ anti-TNF $\alpha-, 0.10$; ADAbs-/anti-TNF $\alpha+, 0.550$.

The probabilities of losing response according to the category of test result were $1.00,0.333,0.500$ and 0.136 , respectively. ADAbs, antidrug antibodies; ; LOR, loss of response; RESP, responders; ; TNF, tumour necrosis factor.

appreciated than alternative test accuracy statistics such as sensitivity and specificity. Negative and positive predictive values vary according to prevalence of the condition being tested for (in this case lack of response). We have meta-analysed the prevalence across the included studies and used this with its $95 \% \mathrm{CI}$ as a guide to the approximate prevalence in which the tests would be performed in practice. The predictive values for each type of test across the relevant prevalence ranges are summarised in figure 6 . As prevalence increases, positive predictive value increases and negative predictive value decreases.

Although pooled prevalence varies somewhat among the four collections of studies, the resulting positive and negative predictive values are similar and range between about $70 \%$ and $80 \%$ implying that between $20 \%$ and $30 \%$

Table 3 Combined assessment of infliximab and antiinfliximab for responders receiving infliximab

\begin{tabular}{|c|c|c|c|c|}
\hline $\begin{array}{l}\text { Imaeda } \\
\text { et } \mathrm{a}^{27}\end{array}$ & ADAbs+ & ADAbs- & Total & $\begin{array}{l}\text { Population } \\
\text { and anti-TNF } \alpha \\
\text { therapy; tests }\end{array}$ \\
\hline \multirow{2}{*}{$\begin{array}{l}\text { Anti- } \\
\text { TNF } \alpha-\end{array}$} & $\mathrm{LOR}=9$ & $\mathrm{LOR}=0$ & $\mathrm{LOR}=9$ & \multirow{6}{*}{$\begin{array}{l}\text { Responders } \\
\text { on infliximab } \\
\text { maintenance; } \\
\text { ELISA. } \\
\text { Prevalence of } \\
\text { LOR=29.3\% }\end{array}$} \\
\hline & $\mathrm{RESP}=1$ & RESP $=7$ & $\mathrm{RESP}=8$ & \\
\hline \multirow{2}{*}{$\begin{array}{l}\text { Anti- } \\
\text { TNF } \alpha+\end{array}$} & $\mathrm{LOR}=3$ & $\mathrm{LOR}=5$ & $\mathrm{LOR}=8$ & \\
\hline & $\mathrm{RESP}=3$ & $\mathrm{RESP}=30$ & $\mathrm{RESP}=33$ & \\
\hline \multirow[t]{2}{*}{ Total } & LOR=12 & $\mathrm{LOR}=5$ & LOR=17 & \\
\hline & $\mathrm{RESP}=4$ & $\mathrm{RESP}=37$ & $\mathrm{RESP}=41$ & \\
\hline
\end{tabular}

The probability of a patient returning each of the four possible test result combinations was:

ADAbs+/anti-TNF $\alpha-$, 0.172; ADAbs+/anti-TNF $\alpha+, 0.103$; ADAbs-/ anti-TNF $\alpha-, 0.121$; ADAbs-/anti-TNF $\alpha+, 0.603$.

The probabilities of losing response according to the category of test result were $0.900,0.500,0.000$ and 0.143 , respectively. ADAbs, antidrug antibodies; ; LOR, loss of response; RESP, responders; TNF, tumour necrosis factor. of positive and negative test results are likely to be incorrect.

In January 2017, we updated our included studies by searching all citations of, and included studies in, five relevant systematic reviews (see online supplement 2 figure 1). ${ }^{6729-31}$ After removal of duplicates and the application of our inclusion criteria, this yielded three ${ }^{32-34}$ and five ${ }^{33} 35-38$ additional studies, respectively, for trough infliximab and trough adalimumab levels (see online supplement 8 table 1). Addition of the former to our meta-analysis had almost no influence on our estimates of test accuracy (see online supplement 8 figures 1 and 2 and table 2); the addition of the adalimumab studies to our meta-analysis also had very little influence on our estimates of test accuracy except a modest reduction in their uncertainty despite doubling the number of available studies (see online supplement 8 figures 1 and 3 and table 3).

\section{DISCUSSION}

The meta-analysis results indicate that the accuracy of tests for predicting lack of response was moderate and that about $20 \%-30 \%$ of both positive and negative test results are likely to be incorrect, with large unexplained heterogeneity between studies. The number of studies on patients treated with adalimumab was too small to draw firm conclusions, but the available evidence suggests similar performance to the tests for infliximab and for antibodies to infliximab.

The sensitivity analyses indicated that much of the variation seen in the forest plots and ROC space could not be explained by our measures of test type and population. Test performance is dependent on cut-offs used for anti-TNF and antibodies to anti-TNF agents and on the time of testing. However, this was not investigated in sensitivity analyses as cut-offs vary by test type as well as within different types of tests, and an agreed cut-off that is transferable between studies and populations has yet to be identified. Furthermore, time of testing was not investigated as all but one study ${ }^{39}$ reported that anti-TNFs levels considered in the studies were trough levels.

Updating the searches found an extra seven studies; however, these made no meaningful difference to the test accuracy estimates. The study designs were largely similar to those in the previous studies. However, there appears to have been a recent waning of interest in antidrug antibodies, possibly attributable to publication of studies indicating their transitory and varying persistence during treatment, while interest in endoscopic healing as an outcome appears to have increased. Additional single arm test accuracy studies may not add significant further understanding in this field. Of more value would be head-to-head test accuracy comparisons in the same population and studies integrating drug levels with other predictive factors to enable more accurate predictions of LOR. 
Table 4 Combined assessment of infliximab and anti-infliximab for people with loss of response receiving infliximab

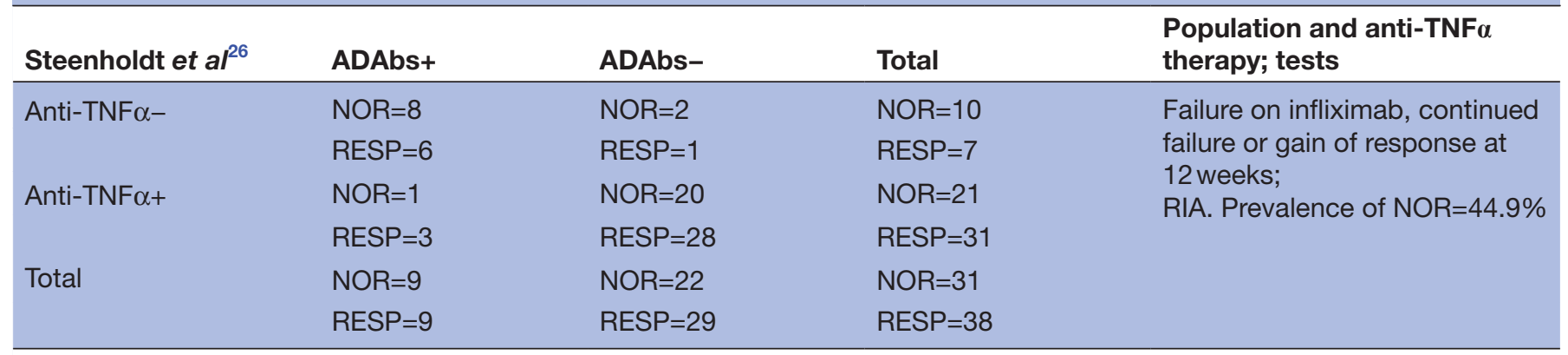

The probability of a patient returning each of the four possible test result combinations was:

ADAbs+/anti-TNF $\alpha-$, 0.203; ADAbs+/anti-TNF $\alpha+, 0.058$; ADAbs-/anti-TNF $\alpha-, 0.00 .043$; ADAbs-/anti-TNF $\alpha+, 0.696$.

The probabilities of failing to gain a response according to category of test result were $0.571,0.250,0.667$ and 0.417 , respectively.

ADAbs, antidrug antibodies; LOR, loss of response; RESP, responders; NOR, no regain of response; TNF, tumour necrosis factor.

Our meta-analyses included studies using different tests for measuring levels of anti-TNF agents and antibodies to anti-TNFs. Although RIA and HMSA tests were used in some of our included studies, the bulk of the tests employed were ELISA tests (26/42, 62\%) encompassing various commercial ELISA kits and ELISAs developed 'in house' by investigators. Several full publications and abstracts have addressed the issue of whether different test methods (eg, solid phase ELISAs, liquid phase assays such as RIA or HMSA) deliver the same quantitative estimates of drug and antibody levels in patient samples. ${ }^{22} 24$ 26-28 40-59 Because there is no consensus about what constitutes a gold standard test, it is difficult to draw conclusions from these studies other than that some differences in performance have been documented. Interestingly, the observed
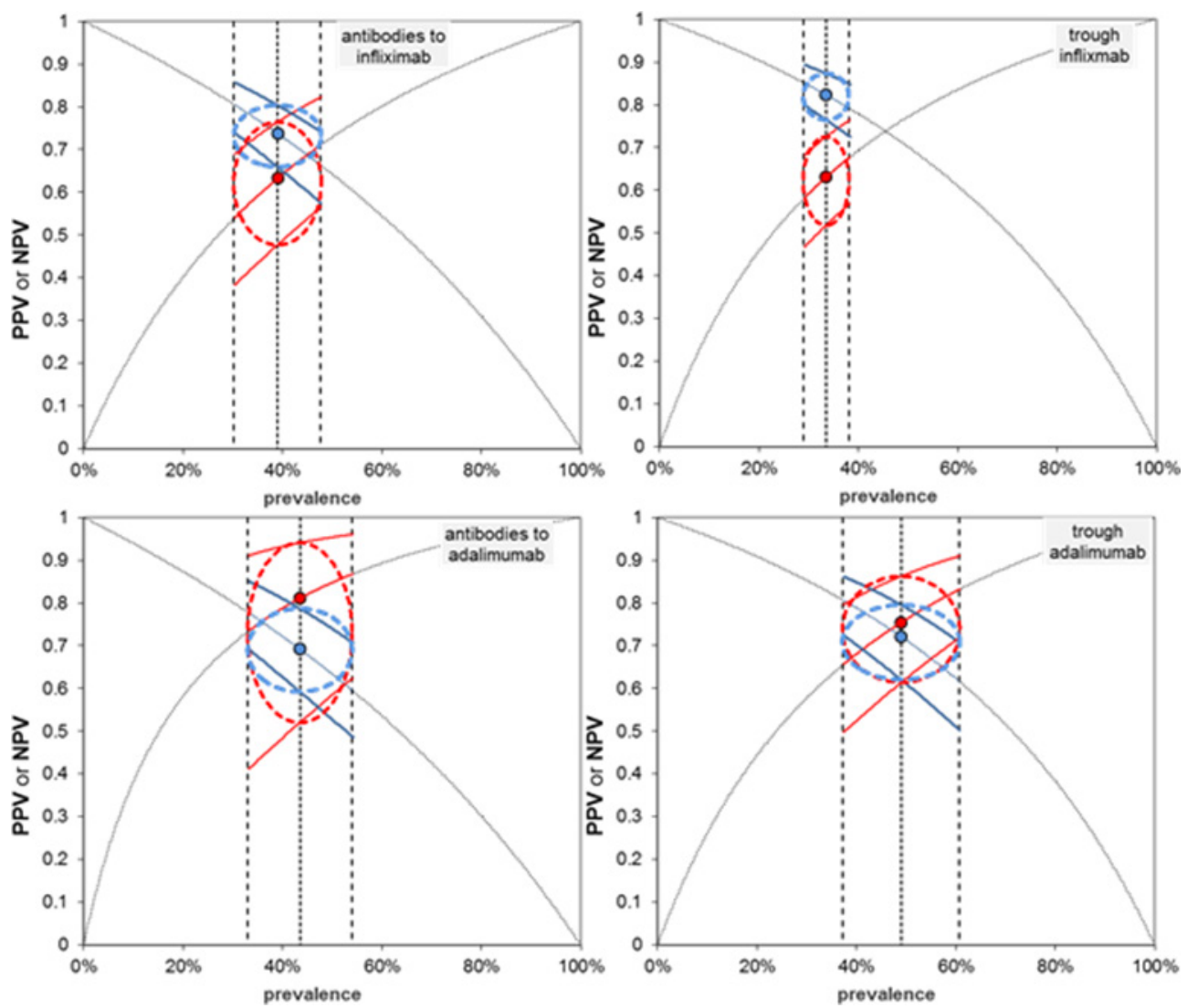

Figure 6 Positive predictive value (PPV) and negative predictive value (NPV) according to the prevalence of lack of response using the pooled summary receiver operating characteristic (sROC) model estimates of sensitivity and specificity. Data points, PPV and NPV at sROC pooled sensitivity and specificity and pooled prevalence; vertical dashed lines, pooled prevalence and 95\% Cls; thick curves, PPV and NPV at upper and lower Cls for sensitivity and specificity across the pooled prevalence and its $95 \% \mathrm{Cl}$. The dashed line ellipses encompass predictive values determined from 95\% Cls of prevalence and $95 \% \mathrm{Cl}$ for PPV and NPV at the point prevalence estimate. 
variation in our meta-analysis could not be explained by the different tests used.

Although the accuracy of the tests for predicting lack of response was found to be moderate, this does not necessarily mean they must lack clinical utility. However, clinicians are likely to be interested in a combined assessment of anti-TNF levels and antibodies to anti-TNF, for which limited accuracy data are available. ${ }^{26-28}$ Diagnostic tests may alter clinical decisions and actions, so evidence beyond test accuracy is required to evaluate clinical value. ${ }^{60}$ Such evidence is best obtained in randomised trials (ie, test and treat investigations), but this is currently sparse. ${ }^{60}$

Two recent randomised controlled trials (RCTs) have compared clinical outcomes between patients whose treatment was directed by algorithms informed by tests for infliximab and/or antibodies to infliximab versus patients who received treatment uninformed by testing. ${ }^{26}{ }^{61}$ In the TAXIT trial, ${ }^{61}$ patients with inflammatory bowel disease responding to infliximab had their dose regimen optimised according to a test algorithm with the aim to bring patients within the therapeutic range and prevent LOR. However, after randomisation to clinically based or test-based dosing, no clinical benefit was observed for patients with $\mathrm{CD}$ at 1 year. Steenholdt et al (2014) ${ }^{26}$ investigated patients who had lost response to infliximab, using a test algorithm to predict the reason for LOR and adjust treatment accordingly. In this equivalence study, no difference in clinical benefit was observed for the test-algorithm group relative to the control group who were prescribed dose intensification. It is notable in this study that for many patients $(14 / 33 ; 42 \%)$, clinicians failed to implement the test-algorithm directive, implying that they may have lacked confidence in the test results or that they considered other factors of overriding importance, as pointed out by Ferrante di Ruffano et al (2012) ${ }^{60}$ Such phenomena (lack of equipoise) complicate assessments of test value. Both of these RCTs reported cost savings in the test-algorithm arm associated with reduced use of infliximab.

This is the first meta-analysis of predictive accuracy of these tests and offers an alternative perspective to earlier meta-analyses. We were able to include more studies than in earlier meta-analyses and have looked at both drug tests and tests for antidrug antibodies and have included studies of patients receiving either infliximab or adalimumab therapies. There was significant heterogeneity between studies, including in the test, outcome measurement and findings, making clinical interpretation difficult.

The meta-analysis results should be viewed with some caution because of the high risk of bias in many of the included studies and because the lack of sufficient numbers of studies precluded subgroup meta-analyses of some types of test (eg, RIA, HMSA).

\section{CONCLUSIONS}

The available evidence suggests that these tests have modest predictive accuracy for clinical status and that about $20 \%-30 \%$ of test results would be likely to be incorrect. However, higher quality head-to-head test accuracy studies are required to enable differentiation between different types of tests and cut-offs, with consistent outcome measurement in the same population. In published trials, the tests have been used for adjusting dose or treatment of patients whose clinical status has already been defined by other criteria. More clinical trial evidence from test-treat studies is required before the clinical utility of the tests can be reliably evaluated.

Contributors KF coordinated the review. RC developed the search strategy and undertook searches. KF, ST-P, MC, AT, DS and PS conducted the clinical effectiveness systematic review, this included: screening and retrieving papers, assessing against the inclusion criteria, appraising the quality of papers and abstracting data from papers for synthesis. MC conducted the data analysis. AC obtained funding, provided project management and methodological advice. RA provided clinical comment and guidance. All authors were involved in writing draft versions of the paper and approved the final version submitted.

Funding This work was commissioned by the NIHR HTA Programme as project number 14/69/03. AC and STP are partly supported by the National Institute for Health Research (NIHR) Collaboration for Leadership in Applied Health Research and Care West Midlands at the University Hospitals Birmingham NHS Foundation Trust.

Competing interests None declared.

Provenance and peer review Not commissioned; externally peer reviewed.

Data sharing statement All data are available from authors on request.

Open Access This is an Open Access article distributed in accordance with the terms of the Creative Commons Attribution (CC BY 4.0) license, which permits others to distribute, remix, adapt and build upon this work, for commercial use, provided the original work is properly cited. See: http://creativecommons.org/ licenses/by/4.0/

(C) Article author(s) (or their employer(s) unless otherwise stated in the text of the article) 2017. All rights reserved. No commercial use is permitted unless otherwise expressly granted.

\section{REFERENCES}

1. Ben-Horin S, Chowers Y. Tailoring anti-TNF therapy in IBD: drug levels and disease activity. Nat Rev Gastroenterol Hepatol 2014;11:243-55

2. Targan SR, Hanauer SB, van Deventer SJ, et al. A short-term study of chimeric monoclonal antibody CA2 to tumor necrosis factor alpha for Crohn's disease. Crohn's Disease cA2 Study Group. N Engl J Med 1997;337:1029-35.

3. Hanauer SB, Sandborn WJ, Rutgeerts P, et al. Human anti-tumor necrosis factor monoclonal antibody (adalimumab) in Crohn's disease: the CLASSIC-I trial. Gastroenterology 2006;130:323-33.

4. Gisbert JP, Panés J. Loss of response and requirement of infliximab dose intensification in Crohn's disease: a review. Am J Gastroenterol 2009;104:760-7.

5. de Boer NKh, Löwenberg M, Hoentjen F. Management of Crohn's disease in poor responders to adalimumab. Clin Exp Gastroenterol 2014;7:83-92.

6. Nanda KS, Cheifetz AS, Moss AC. Impact of antibodies to infliximab on clinical outcomes and serum infliximab levels in patients with inflammatory bowel disease (IBD): a meta-analysis. Am J Gastroenterol 2013;108:40-7.

7. Paul S, Moreau AC, Del Tedesco E, et al. Pharmacokinetics of adalimumab in inflammatory bowel diseases: a systematic review and meta-analysis. Inflamm Bowel Dis 2014;20:1288-95.

8. Garcês S, Demengeot J, Benito-Garcia E. The immunogenicity of anti-TNF therapy in immune-mediated inflammatory diseases: a systematic review of the literature with a meta-analysis. Ann Rheum Dis 2013;72:1947-55

9. Lee LY, Sanderson JD, Irving PM. Anti-infliximab antibodies in inflammatory bowel disease: prevalence, infusion reactions, immunosuppression and response, a meta-analysis. Eur $\mathrm{J}$ Gastroenterol Hepatol 2012;24:1078-85.

10. Pepe NS. The statistical evaluation of medical tests for classification and prediction. New York: Oxford University Press, 2003. 
11. Whiting PF, Rutjes AW, Westwood ME, et al. QUADAS-2: a revised tool for the quality assessment of diagnostic accuracy studies. Ann Intern Med 2011;155:529-36.

12. Harbord RM, Deeks JJ, Egger M, et al. A unification of models for meta-analysis of diagnostic accuracy studies. Biostatistics 2007;8:239-51.

13. Harbord R, Whiting P. Metandi: meta-analysis of diagnostic accuracy using hierarchical logistic regression. Stata Journal 2009;9:211-29.

14. Bossuyt PM, Davenport C, Deeks JJ, et al; Chapter 11: interpreting results and drawing conclusions. In: Deeks JJ, Bossuyt PM, Gatsonis $\mathrm{C}$, eds. Cochrane handbook for systematic reviews of diagnostic test accuracy version 09: the Cochrane collaboration, 2013.

15. Wallace BC, Schmid CH, Lau J, et al. Meta-Analyst: software for meta-analysis of binary, continuous and diagnostic data. BMC Med Res Methodol 2009;9:80.

16. Deeks JJ, Macaskill P, Irwig L. The performance of tests of publication bias and other sample size effects in systematic reviews of diagnostic test accuracy was assessed. J Clin Epidemiol 2005;58:882-93.

17. Ben-Bassat $O$, Romanova A, lacono A et al. Tu1150 Association of serum infliximab and antibodies to infliximab to long-term clinical outcome and mucosal healing in Crohn's Disease. Gastroenterology 2013:144(5 Suppl):S-775.

18. Yanai H, Mlynarsky L, Ron Y, et al. Tu1267 the questionable value of infliximab trough levels during prolonged maintenance therapy. Gastroenterology 2012;142:S-788-0.

19. Kong JY, Bundell CS, Pawlik J, et al. Trough serum infliximab level, anti-infliximab antibody status and response to infliximab maintenance treatment in inflammatory bowel disease (IBD). $J$ Gastroenterol Hepatol 2011;26:59-60.

20. Marzo M, Armuzzi A, Felice C, et al. P.06.20 role of trough levels and antibodies to infliximab in the evaluation of loss of response and infusion reactions to infliximab therapy in inflammatory bowel disease. Digestive and Liver Disease 2014;46:S77.

21. Nagore D, del Agua AR, Pascual J, et al. Tu1325 Therapeutic cut-off of infliximab in patients with inflammatory bowel diseases. Gastroenterology 2015;148(4 suppl 1):S-860.

22. Bodini G, Savarino V, Dulbecco P, et al. P505 ELISA vs. HMSA: a comparison between two different methods for the evaluation of adalimumab serum concentration and anti-adalimumab antibodies preliminary data. J Crohn's Colitis 2014;8:S278.

23. Dauer RM, Yarur AJ, Abreu MT. Su1216 Infliximab re-induction outcomes after a failure to treatment. Gastroenterology 2013;144(5 Suppl):S-430.

24. Vande Casteele N, Gils A, Singh S, et al. Antibody response to infliximab and its impact on pharmacokinetics can be transient. Am J Gastroenterol 2013;108:962-71

25. Hanauer SB, Wagner CL, Bala M, et al. Incidence and importance of antibody responses to infliximab after maintenance or episodic treatment in Crohn's disease. Clin Gastroenterol Hepatol 2004;2:542-53.

26. Steenholdt C, Brynskov J, Thomsen OØ, et al. Individualised therapy is more cost-effective than dose intensification in patients with Crohn's disease who lose response to anti-TNF treatment: a randomised, controlled trial. Gut 2014;63:919-27.

27. Imaeda $\mathrm{H}$, Andoh A, Fujiyama Y. Development of a new immunoassay for the accurate determination of anti-infliximab antibodies in inflammatory bowel disease. $J$ Gastroenterol 2012;47:136-43.

28. Imaeda $\mathrm{H}$, Takahashi $\mathrm{K}$, Fujimoto $\mathrm{T}$, et al. Clinical utility of newly developed immunoassays for serum concentrations of adalimumab and anti-adalimumab antibodies in patients with Crohn's disease. $J$ Gastroenterol 2014;49:100-9.

29. Barnes EL, Allegretti JR. Are Anti-Tumor necrosis factor trough levels predictive of mucosal healing in patients with inflammatory bowel disease?: a systematic review and meta-analysis. J Clin Gastroenterol 2016;50:733-41.

30. Moore C, Corbett G, Moss AC. Systematic review and meta-analysis: serum infliximab levels during maintenance therapy and outcomes in inflammatory bowel disease. J Crohns Colitis 2016;10:619-25.

31. Silva-Ferreira F, Afonso J, Pinto-Lopes P, et al. A systematic review on infliximab and adalimumab drug monitoring: levels, clinical outcomes and assays. Inflamm Bowel Dis 2016;22:2289-301.

32. Levesque BG, Greenberg GR, Zou G, et al. A prospective cohort study to determine the relationship between serum infliximab concentration and efficacy in patients with luminal Crohn's disease. Aliment Pharmacol Ther 2014;39:1126-35.

33. Ungar B, Levy I, Yavne Y, et al. Optimizing anti-TNF- $\alpha$ therapy: serum levels of infliximab and adalimumab are associated With mucosal healing in patients with inflammatory bowel Diseases. Clin Gastroenterol Hepatol 2016;14:550-7.
34. Reinisch W, Reinink AR, Higgins PD. Factors associated with poor outcomes in adults with newly diagnosed ulcerative colitis. Clin Gastroenterol Hepatol 2015;13:635-42.

35. Bodini G, Giannini EG, Furnari M, et al. Comparison of two different techniques to assess adalimumab trough levels in patients with Crohn's Disease. J Gastrointestin Liver Dis 2015;24:451-6.

36. Zittan E, Kabakchiev B, Milgrom R, et al. Higher adalimumab drug levels are associated with mucosal healing in patients with Crohn's Disease. J Crohns Colitis 2016;10:510-5.

37. Yarur AJ, Jain A, Hauenstein SI, et al. Higher adalimumab levels are associated with histologic and endoscopic remission in patients with Crohn's Disease and Ulcerative Colitis. Inflamm Bowel Dis 2016;22:409-15.

38. Morita $\mathrm{Y}$, Imaeda $\mathrm{H}$, Nishida A, et al. Association between serum adalimumab concentrations and endoscopic disease activity in patients with Crohn's disease. J Gastroenterol Hepatol 2016;31:1831-6.

39. Chiu YL, Rubin DT, Vermeire $S$, et al. Serum adalimumab concentration and clinical remission in patients with Crohn's disease. Inflamm Bowel Dis 2013;19:1112-22.

40. Kopylov U, Mazor Y, Yavzori M, et al. Clinical utility of antihuman lambda chain-based enzyme-linked immunosorbent assay (ELISA) versus double antigen ELISA for the detection of anti-infliximab antibodies. Inflamm Bowel Dis 2012;18:1628-33.

41. Corstjens PL, Fidder HH, Wiesmeijer KC, et al. A rapid assay for on-site monitoring of infliximab trough levels: a feasibility study. Anal Bioanal Chem 2013;405:7367-75.

42. Steenholdt C, Ainsworth MA, Tovey M, et al. Comparison of techniques for monitoring infliximab and antibodies against infliximab in Crohn's disease. Ther Drug Monit 2013;35:530-8.

43. Vande Casteele N, Buurman DJ, Sturkenboom MG, et al. Detection of infliximab levels and anti-infliximab antibodies: a comparison of three different assays. Aliment Pharmacol Ther 2012;36:765-71.

44. Wang SL, Ohrmund L, Hauenstein S, et al. Development and validation of a homogeneous mobility shift assay for the measurement of infliximab and antibodies-to-infliximab levels in patient serum. J Immunol Methods 2012;382:177-88.

45. Steenholdt C, Bendtzen K, Brynskov J, et al. Clinical implications of measuring drug and anti-drug antibodies by different assays when optimizing infliximab treatment failure in Crohn's disease: post hoc analysis of a randomized controlled trial. Am J Gastroenterol 2014;109:1055-64.

46. Ruiz-Argüello B, del Agua AR, Torres N, et al. Comparison study of two commercially available methods for the determination of infliximab, adalimumab, etanercept and anti-drug antibody levels. Clin Chem Lab Med 2013;51:e287-9.

47. Daperno M, Frigerio F, Guiotto C, et al. P508 evaluation of the diagnostic performance of two commercially available tests for infliximab trough levels (IFX-TL) and antibodies to infliximab (ATI) titration in inflammatory bowel disease (IBD). J Crohn's and Colitis 2013;7:S213-4.

48. Egea-Pujol L, Reddy R, Patel S, et al. Homogenous mobility shift assay (HMSA) overcomes the limitations of ELISA and ECLIA assays for monitoring infliximab (IFX), adalimumab (ADA), and associated anti-drug antibodies in serum. Am J Gastroenterol 2013;108:S548.

49. Eser A, Primas C, Hauenstein S, et al. Tu1164 comparison of early measurement of Infliximab and antibodies-to-infliximab serum levels with standard trough analysis. Gastroenterology 2013;144(5 Suppl):S-779.

50. Eser A, Primas C, Haunstein S, et al. P551 detection of anti infliximab antibodies in patients with inflammatory bowel disease (IBD) in the presence of infliximab by homogeneous liquid phase anti infliximab mobility shift assay. J Crohn's and Colitis 2013;7:S231-2.

51. Greathead L, Kelleher P, Steel A. P086 Development and validation of ELISA to measure serum anti TNFa levels. J Crohn's and Colitis 2014;8:S97-S98

52. Hauenstein S, Ohrmund L, Salbato J, et al. Su1928 comparison of homogeneous mobility shift assay and solid phase ELISA for the measurement of drug and anti-drug antibody (ADA) levels in serum from patients treated with anti-TNF biologics. Gastroenterology 2012;142:S-538.

53. McTigue M, Sandborn W, Levesque B, et al. Clinical utility of next generation infliximab and antibodies to infliximab assay. Am J Gastroenterol 2013;108:S527.

54. Imaeda $\mathrm{H}$, Andoh A, Fujiyama Y. Development of a new immunoassay for the accurate determination of anti-infliximab antibodies in inflammatory bowel disease. J Gastroenterol 2012;47:136-43.

55. Ungar B, Anafy A, Kopylov U, et al. Sa1258 the clinical and immunological significance of low level of Infliximab in the absence 
of Anti-Infliximab antibodies in patients with IBD. Gastroenterology 2014;146(5 Suppl):S-245

56. Casteele NV, Peeters M, Compernolle G, et al. Sa1248 TNFresponsive cellular based assay reveals neutralizing capacity of anti-adalimumab antibodies in Crohn's Disease and ulcerative colitis patients. Gastroenterology 2014;146(5 Suppl):S-242.

57. Wang S-L, Ohrmund L, Singh S. W1256 measurement of human anti-chimeric antibodies (Haca) and Infliximab levels in patient serum using a novel homogeneous assay. Gastroenterology 2010;138:S-684-0.

58. Schatz SB, Prell C, Freudenberg F, et al. PA-G-0035 comparison of different tests for determination of infliximab levels and antibodies against infliximab in pediatric IBD patients. The 46th annual meeting of the European Society of Paediatric Gastroenterology, Hepatology and Nutrition. J Pediatr Gastroenterol Nutr 2013;56(suppl 2):19

59. Wang SL, Ohrmund L, Hauenstein S, et al. Evaluation of a novel homogeneous mobility shift assay for the measurement of human antibodies-To-Infliximab and infliximab levels in patient serum. Am J Gastroenterol 2011;106:S475-6.

60. Ferrante di Ruffano L, Hyde CJ, McCaffery KJ, et al. Assessing the value of diagnostic tests: a framework for designing and evaluating trials. BMJ 2012;344:e686.

61. Vande Casteele N, Ferrante M, Van Assche G, et al. Trough concentrations of infliximab guide dosing for patients with inflammatory bowel disease. Gastroenterology 2015;148:1320-9.

62. Ben-Horin S, Mazor Y, Yanai H, et al. The decline of anti-drug antibody titres after discontinuation of anti-TNFs: implications for predicting re-induction outcome in IBD. Aliment Pharmacol Ther 2012;35:714-22.

63. Candon S, Mosca A, Ruemmele F, et al. Clinical and biological consequences of immunization to infliximab in pediatric Crohn's disease. Clin Immunol 2006;118:11-19.

64. Pariente B, Pineton de Chambrun G, Krzysiek R, et al. Trough levels and antibodies to infliximab may not predict response to intensification of infliximab therapy in patients with inflammatory bowel disease. Inflamm Bowel Dis 2012;18:1199-206.

65. Baert F, Drobne D, Gils A, et al. Early trough levels and antibodies to infliximab predict safety and success of reinitiation of infliximab therapy. Clin Gastroenterol Hepatol 2014;12:1474-81.

66. Ainsworth MA, Bendtzen K, Brynskov J. Tumor necrosis factoralpha binding capacity and anti-infliximab antibodies measured by fluid-phase radioimmunoassays as predictors of clinical efficacy of infliximab in Crohn's disease. Am J Gastroenterol 2008;103:944-8.

67. Farrell RJ, Alsahli M, Jeen YT, et al. Intravenous hydrocortisone premedication reduces antibodies to infliximab in Crohn's disease: a randomized controlled trial. Gastroenterology 2003;124:917-24.

68. Steenholdt C, Palarasah Y, Bendtzen K, et al. Pre-existing IgG antibodies cross-reacting with the Fab region of infliximab predict efficacy and safety of infliximab therapy in inflammatory bowel disease. Aliment Pharmacol Ther 2013;37:1172-83.

69. Steenholdt C, Bendtzen K, Brynskov J, et al. Cut-off levels and diagnostic accuracy of infliximab trough levels and anti-infliximab antibodies in Crohn's disease. Scand J Gastroenterol 2011;46:310-8.

70. Ben-Horin S, Yavzori M, Katz L, et al. The immunogenic part of infliximab is the $F\left(a b^{\prime}\right) 2$, but measuring antibodies to the intact infliximab molecule is more clinically useful. Gut 2011;60:41-8.

71. Mazor Y, Almog R, Kopylov U, et al. Adalimumab drug and antibody levels as predictors of clinical and laboratory response in patients with Crohn's disease. Aliment Pharmacol Ther 2014;40:620-8.

72. Roblin X, Marotte H, Rinaudo M, et al. Association between pharmacokinetics of adalimumab and mucosal healing in patients with inflammatory bowel diseases. Clin Gastroenterol Hepatol 2014;12:80-4.

73. Frederiksen MT, Ainsworth MA, Brynskov J, et al. Antibodies against infliximab are associated with de novo development of antibodies to adalimumab and therapeutic failure in infliximab-to-adalimumab switchers with IBD. Inflamm Bowel Dis 2014;20:1714-21.

74. West RL, Zelinkova Z, Wolbink GJ, et al. Immunogenicity negatively influences the outcome of adalimumab treatment in Crohn's disease. Aliment Pharmacol Ther 2008;28:1122-6.

75. Bortlik M, Duricova D, Malickova K, et al. Infliximab trough levels may predict sustained response to infliximab in patients with Crohn's disease. J Crohns Colitis 2013;7:736-43.

76. Cornillie F, Hanauer SB, Diamond RH, et al. Postinduction serum infliximab trough level and decrease of C-reactive protein level are associated with durable sustained response to infliximab: a retrospective analysis of the ACCENT I trial. Gut 2014;63:1721-7.

77. Hibi T, Sakuraba A, Watanabe M, et al. C-reactive protein is an indicator of serum infliximab level in predicting loss of response in patients with Crohn's disease. J Gastroenterol 2014;49:254-62.

78. Maser EA, Villela R, Silverberg MS, et al. Association of trough serum infliximab to clinical outcome after scheduled maintenance treatment for Crohn's disease. Clin Gastroenterol Hepatol 2006;4:1248-54. 